\title{
Research on Wireless Sensor Network Localization Technology Based on Range-Free positioning technology
}

\author{
Yunsai Zhai ${ }^{1, a}$, Lichen Zhang ${ }^{2, b}$ \\ ${ }^{1}$ Faculty of Computer Science and Technology, Guangdong University of Technology, \\ Guangzhou, 510090, China \\ ${ }^{2}$ Faculty of Computer Science and Technology, Guangdong University of Technology, \\ Guangzhou, 510090, China \\ aemail: zys_yunsai@163.com, bemail: zhanglichen1962@163.com
}

Keywords: WSN; Range-Based; Range-Free; DV-HOP

\begin{abstract}
Wireless sensor network localization technology is currently a hot research topic. WSN is a special self-organizing network, Range-free positioning technology at the advantages of its low cost and its simple hardware equipment has been widely concerned by the researchers. Therefore, this paper studies the 6 typical Range-free algorithm, then analyzes DV-HOP positioning algorithm of the Range-free positioning technology. Finally, the work of the paper is summarized and the future work is prospected.
\end{abstract}

\section{Introduction}

Wireless sensor network[1] is called WSN for short. Wireless sensor network is composed of a large number of cheap micro sensor nodes that these cheap micro sensor nodes are deployed in the monitoring region. WSN, through a multi hop ad hoc network system formed by wireless communication, bring a change of information perception with its low power consumption, low cost, distributed and self-organization characteristics. Its purpose is to perceive, collect and process the information of the perceived object in the network coverage area, and send it to the observer. Sensors, sensing objects and observers constitute have formed the three elements of the wireless sensor network. Wireless sensor network is the rapid development of high technology for a regional information collection, transmission, analysis and processing in recent years. Wireless sensor networks in military applications, environmental monitoring and disaster warning, medical monitoring, geological exploration and other fields have broad application prospects.

\section{Wireless sensor network positioning technology}

The positioning technology is not only the high technology which is a kind of widely used, but also important supporting technology of WSN, and it has a wide range of applications. Wireless sensor network as a new information acquisition and processing technology, in target tracking, intrusion detection and some location related fields (such as routing based on location information, intelligent transportation, logistics management, and many other applications), has a broad application prospects. However, whether in military reconnaissance or geographic environmental monitoring, or traffic condition detection or tracking of patients in health care or many applications occasions, a lot of gained monitoring information need attach the corresponding location information, even meticulous to the application of some of our mobile terminals. If there is no location information, data collected by the sensor nodes is almost no application value. Because the sensor nodes must first clear its position to detail " what happened in this position", thus to realize the external target locating and tracking. So the self-localization of the sensor nodes in the network is foundation of sensor network applications.

The positioning of wireless sensor networks has become a hot research topic, positioning method of wireless sensor network based on distance measurement and it can be divided into[2][3]: ranging algorithm and non-ranging algorithm. (1) range-based: It is positioning algorithm based on Ranging 
Technology, measures point-to-point distance between nodes or angle information and uses the three side measurement method, the triangulation method or the maximum likelihood estimation method to calculate node location. In location algorithm, positioning nodes uses a certain distance measurement technology to measure the distance to anchor node, usually takes at least three ranging information and uses three side measurement algorithm for calculating position. Common ranging technology has signal strength (RSSI), time difference of arrival (TOA) and so on. Positioning accuracy of ranging algorithm is relatively high, but increasing the cost and energy consumption, is more sensitive to the ranging error. (2) range-free: It do not need no distance and angle information, only according to the network connectivity and other information to achieve node localization, reduce costs, and at the same time sacrifice the positioning accuracy.

The positioning algorithm based on Ranging Technology needs to measure the absolute distance or direction between adjacent nodes, and use the actual distance between nodes to calculate the distance between unknown nodes. The positioning algorithm based on Range-free Technology does not need this measurement information, it is based on the network connectivity and other information, and uses the estimated distance between nodes to compute the unknown node. The precision of ranging method is generally higher than that of non-ranging method, but ranging method to the hardware requirement of the node are higher, in certain situations, such as in a network with large scale and sparse anchor nodes, when the location node is unable to direct communication with enough anchor nodes, common ranging method is difficult to locate, at this time you need to consider use non ranging way to estimate the distance between nodes. Two kinds of algorithm have its own limitations. The Range-free's positioning algorithm has the advantages of low cost, low power consumption, low noise resistance, simple hardware equipment and so on. At this stage, more research is the Range-free's positioning algorithm, so there is no need to add extra hardware, can also reduce the volume of the sensor nodes.

\section{6 kind of typical non-ranging positioning algorithm}

Difference between non-ranging algorithm and ranging algorithm depends on the fact that the former does not directly measure the distance, but uses connectivity of the network to estimate the distance or the coordinate from the node to the anchor node. Due to the uncertainty of the method, methods based on non-ranging algorithm are so many. The algorithm generally uses the ratio of error value to wireless communication radius of nodes to represent the positioning accuracy of sensor nodes. As long as the positioning accuracy is less than $40 \%$, it can satisfy the requirements of most applications. According to the time sequence, this paper introduces some typical non-ranging algorithm[4].

Bulusu (et al.) proposed a single hop, low power algorithm, which uses the anchor node connectivity to determine the coordinates. The coordinates of unknown nodes are obtained by calculating the centroid of all nodes in the radio range. Nodes locate themselves in the center of mass of the nodes that are close to them. The algorithm, although simple, but error is large, the need of anchor node density is high. About $90 \%$ of the nodes positioning accuracy is within $1 / 3$ of the anchor node distribution interval.

He (et al.) proposed APIT algorithm, target node selected randomly three adjacent anchor nodes and tested whether the unknown nodes are located in a triangle which are formed by these unknown nodes. Different anchor nodes are combined and retested, until they exhausted all combinations or reach the required positioning accuracy. Finally, the algorithm calculates the intersection centroid of all triangles that contain the target node, and takes it as the target node position. The algorithm needs a higher anchor node density, and its positioning accuracy is $40 \%$.

Niculescu (et al.) proposed DV-hop localization algorithm, it collects information from neighboring nodes in the network, and calculates the shortest path between non adjacent nodes. DV-Hop algorithm uses the coordinates of known position nodes to estimate a jump distance and uses to the jump distance of the shortest path to estimate the distance of the unknown node and anchor node. The algorithm is only for dense network of isotropic, when the density of anchor 
nodes is $10 \%$, the positioning error is $33 \%$.

Radhika (et al.) proposed Amorphous Positioning algorithm, it uses off-line jump distance to estimate, same as DV-Hop algorithm, going through a neighboring node information exchange to improve estimated value of location. It needs to predict the connectivity of the network, when the network connectivity is 15 , the positioning accuracy is $20 \%$.

Savvides (et al.) introduced a N-Hop multilateration algorithm. It uses Calman filtering technique to achieve iteratively refinement. The algorithm can avoid the error accumulation caused by multi hop transmission in sensor networks and improve the accuracy, the node communication distance is $15 \mathrm{~m}$. When the anchor node density is $20 \%$ and ranging error is $1 \mathrm{~cm}$, the positioning error is $3 \mathrm{~cm}$.

Capkun (et al.) proposed self-positioning algorithm (SPA). The algorithm firstly establishes local coordinate system between each node according to the scope of the communication, and then establishes global coordinate system through information exchange and coordination between nodes. The node in the network can calculate its position in the coordinate system which is established by the nodes which are separated by $\mathrm{N}$ hop .

In conclusion, its non-ranging algorithms are mostly theoretical research, and are closely related to the connectivity of the network and the density of nodes. Therefore, its scope of application has certain limitations, in the process of wireless sensor network positioning technology research, we should be more consideration range-based location algorithm.

\section{DV-HOP location algorithm}

A more classical non-ranging algorithm, such as centroid algorithm proposed by Belusu and Heidemann, calculate position according to the received anchor node position information. DVHOP algorithm[5][6] is based on the hop count to anchor node to calculate position, and then calculate the position of the three side measurements, in which it uses RSSI technology to increase the DV-HOP positioning accuracy.

DV-HOP algorithm is a kind of localization algorithm without distance measurement, which is proposed by Dragos Niculescu using distance vector routing and GPS positioning principle. Because the sensor node communication distance is limited, they often transmit data through the way of multi hop routing, and the node itself only exchanges information with adjacent nodes, DV-HOP algorithm uses this feature. The basic principle of the DV-HOP algorithm is to use the product of Network average hop distance and the shortest hop between unknown nodes and anchor nodes to represent the distance between the unknown node and the anchor node. Although the number of reference nodes in the communication range of the node to be located is not much, using the above method can get estimated distance of multiple reference nodes outside the communication range, the above method uses a large amount of redundant information to realize node localization. The algorithm first uses distance vector exchange protocol to make all nodes in the network get the hop count to the anchor node, and then the anchor node calculates the network average distance per hop and broadcast it to the network. When the unknown node receives the value, it can calculate the distance between unknown node and anchor node according to hop count. When the unknown nodes obtain the distance of 3 or more than 3 anchor nodes, then it can perform three (multiple) side measurements or a maximum likelihood estimation method.

Compared with the range-based positioning technology, the accuracy of DV-HOP algorithm is low, but DV-HOP algorithm does not need the nodes have ranging capability, it requires no additional hardware, its energy consumption is low and it is less affected by the environment. Its algorithm is simple and it is easy to implement. For isotropic dense network, it can get reasonable average distance per hop, and can meet the requirements of most applications in positioning accuracy, etc. It is more suitable for large scale and low energy of WSN in the Hardware size and power consumption. It is positioning mechanism of much attention at present, many positioning systems have been implemented by this algorithm. 


\section{Conclusions}

The practicality of most range-based localization algorithm practical is very poor, it just stays in the stage of theoretical research and is mostly carried out in the simulation environment. It needs to assume a lot of uncertain factors, and these factors cannot be satisfied in practical application, so these algorithms lost practical significance. Therefore, design of positioning algorithm should be more from the practical application consideration, we should design a practical location algorithm based on the practical application.

\section{Acknowledgment}

In this paper, the research was sponsored by the Guangdong Natural Science Foundation under grant (Project No. 2015A030313490), all support is gratefully acknowledged.

\section{References}

[1] Linxing Chen. Wireless sensor network technology and application [M]. Electronics Industry Publishing House, 2009.

[2] Jianguo Wang, Lin Zhang. Research on Localization Technology of wireless sensor network node based on non-ranging [J]. Computer Development and Application, 2009, 22 (11): 52-54.

[3] Zhifa Shen. Research on Localization Algorithm of wireless sensor network node based on non-ranging [D]. Chongqing University of Posts and Telecommunications, 2011.

[4] Wei Lin, Chuanfeng Chen. Based on RSSI wireless sensor network triangle centroid location algorithm [J]. Modern Eectronic Technology, 2009, 32 (2): 180-182.

[5] YanNi Hai, XiangYue Ying, Gavin Jane. An improved algorithm for wireless sensor based on DV-Hop [J]. Industrial Control Computer, (12): 43-45.

[6] Ji Cao. Research on Localization Algorithm of wireless sensor networks nodes based on non-ranging [D]. Jiangxi University of Science and Technology, 2015. 